Check for updates

Cite this: RSC Adv., 2019, 9, 32683

Received 6th May 2019

Accepted 17th August 2019

DOI: $10.1039 / c 9 r a 03365 c$

rsc.li/rsc-advances

\section{Strategically prolonged release of multiple antibacterial components from a thin film coating for synergistic effects against Staphylococcus aureus infections}

\begin{abstract}
Meijun Song, $\dagger^{\text {ab }}$ Haidong Zhu, $\dagger^{c}$ Lin Ye, ${ }^{d}$ Chengxiang $L_{i u}^{d}$ and Zhaojun Xu*e
Biomaterial-associated infections (BAIs) remain a major challenge in clinical surgery because they can potentially cause serious disabilities in patients. This study investigated the use of a multilayer coating technology that can co-deliver two therapeutic components, gentamicin and OP-145 peptide, to treat Staphylococcus aureus effectively. A biocompatible and biodegradable thin film was produced via layerby-layer assembly using an antibacterial peptide and gentamicin. The thin film was systematically characterized, showing controllable features such as thickness, transparency, cargo loading, and cargo release. In vitro tests showed that the thin film has fewer toxicity problems than soluble cargos; compared to cargos in a soluble form, the thin film has minor impacts on mammalian cells' metabolism. Additionally, the antibacterial cargos assembled on the thin film can effectively inhibit the formation of biofilms and show effective in vitro antibacterial potency. In vivo studies illustrated that the thin film can inhibit the progression of $S$. aureus in a mouse model, indicating the effectiveness of the thin film structure in the clinic. This study demonstrates that a thin film composed of gentamicin and OP-145 could be employed to prevent BAls in clinical surgery.
\end{abstract}

\section{Introduction}

One challenge in clinical surgery is biomaterial-associated infections (BAIs), which can lead to serious disabilities in patients. ${ }^{1}$ Two major causes of BAIs are Staphylococcus aureus or $S$. epidermidis infections. These bacteria can generate aggregates or biofilms on the surfaces of implants and progress into the extracellular matrix of the implants. The bacteria have low metabolic activity, making traditional antibiotics less effective against them. Infections by these bacteria can thus progress within the cellular matrix, leading to long-term chronic infections that may eventually cause disabilities in patients. ${ }^{2}$ This issue is even more challenging because the residues of the bacteria may remain even after the implants have been removed. ${ }^{3,4}$ For example, existing human pathological studies

${ }^{a}$ Department of Respiratory Medicine, Zhejiang Provincial People's Hospital, Hangzhou, China, 310016

${ }^{b}$ Department of Infectious Diseases, Sir Run Run Shaw Hospital, College of Medicine, Zhejiang University, Hangzhou, China, 310016

'Department of Radiology, Ningbo Medical Center, Lihuili Eastern Hospital, Ningbo, Zhejiang, China, 315000

${ }^{d}$ Department of Emergency Medicine, Ningbo No. 2 Hospital, Ningbo, Zhejiang, China, 315000

${ }^{e}$ Department of Intensive Care Unit, Ningbo No. 2 Hospital, Ningbo, Zhejiang, China, 315000. E-mail: smjrebecca@163.com; Tel: +8615267096922

$\dagger$ These authors contribute equally to this work. indicated that 15 out of 44 prosthetic joints in patients were associated with $S$. aureus infection during the surgery or even the entire life span of the implants. ${ }^{5}$ Another study showed that patients with orthopedic implants have a greater chance of $S$. aureus infection relapse compared to the patients that received no implants. ${ }^{6}$

Biomedical engineering-enabled strategies may help solve these challenges due to their unique possibilities, including the controlled release and co-delivery of therapeutic components at the implanted tissue area. ${ }^{-9}$ For example, clinical studies have been using the controlled release of antibiotics such as gentamicin and doxycycline from coatings to protect tissues from infections. ${ }^{10}$ However, antibiotics are being overused around the world, especially in developing countries, making the future efficacy of existing antibiotics uncertain. ${ }^{\mathbf{1 1 , 1 2}}$ Thus, novel technologies, especially bioengineering-based technologies, need to be developed to prevent BAI-related bacterial infections.

Several therapeutic cargos, including small molecules and peptides, have been used to prevent $S$. aureus in the clinic. Among these drugs, gentamicin has attracted considerable attention due to its broad antibacterial spectrum. ${ }^{9}$ In addition to gentamicin, antimicrobial peptides with antimicrobial functions are broadly investigated for treating infection diseases. Current studies showed that natural antibacterial peptide such as human cathelicidin LL-37 had a wide antimicrobial spectrum, and was able to eradicate multidrug-resistant 
bacteria. $^{\mathbf{1 3 , 1 4}}$ Compared to conventional antibiotics, these peptides are more potent than conventional antibiotics in combating multi-drug resistant and biofilm infections. ${ }^{15,16}$ These peptides are mostly small in size and charged in nature, so they can interact with the bacterial membrane, causing bacterial lysis within several minutes. ${ }^{15-17}$ Based on human cathelicidin LL-37, researchers has developed synthetic antibacterial peptide OP-145. Existing studies showed that OP-145 can effectively reduce drug-resistant Staphylococcus aureus and inhibit the formation of the formation of biofilm. ${ }^{13,14}$ Thus, these attractive features make the peptides very promising for treating BAI-related infections.

Anti-infective coating has been employed as a "contact killing" and antimicrobial technology in for implanting different devices. ${ }^{\mathbf{1 8}, 19}$ Depending on the features, the coating could be degraded or be not degraded in the in vivo environment. The coating technology could also be designed to be "smart", allowing controlled release of antibacterial components in a time or environmental-responsive manner. ${ }^{18,19}$ This study aimed to use a coating technology (i.e., layer-by-layer assembly) to create functional thin films that can co-deliver therapeutic components for treating $S$. aureus effectively. The thin film will be used to prevent potential antibiotic resistance issues in the clinic. Toward this goal, the technology was designed to load controllable levels of therapeutic cargos that allow the eradication of bacteria and inhibit the formation of bacterial biofilms. Specifically, we used a biocompatible, biodegradable thin film composed of polyelectrolyte multilayers (PEMs). ${ }^{20,21}$ The PEMs were assembled by depositing oppositely charged materials in a layer-by-layer manner. The PEMs formed by this technology are simple to manufacture, tunable in composition, flexible in cargo loading and controllable in cargo release. One of the major advantages of PEMs is that they allow the incorporation of multiple therapeutic components; thus, they can potentially overcome drug resistance issues by employing the synergistic effects offered by the simultaneous use of different cargos. Existing studies have examined PEMs for controlled-release therapeutic cargos, including painkilling or anti-inflammatory drugs, peptides, and antibiotics. ${ }^{22-25}$ The major objective of this work is to construct and characterize PEM films that contain two different therapeutic cargos and to assess the antibiotic functions of the PEMs against $S$. aureus infections.

\section{Materials and methods}

\section{Materials}

A penicillin/streptomycin solution, gentamicin sulfate, $70 \% \mathrm{v} / \mathrm{v}$ isopropyl alcohol, phosphate-buffered saline (PBS) were purchased from Sigma-Aldrich (Shanghai, China). N-type silicon wafers were obtained from Silicon Quest (Santa Clara, CA). The peptide OP-145, with a sequence of acetylIGKEFKRIVERIKRFLRELVRPLR-amide, was obtained from Shanghai Apeptide Co. Ltd. (Shanghai, China). Mouse preosteoblast cells (MC3T3-E1 Subclone 4 CRL-2594) were originally purchased from ATCC (Manassas, VA). Cell culture materials, such as alpha minimum essential medium ( $\alpha$-MEM), fetal bovine serum (FBS), and trypsin-EDTA, were from
Invitrogen (Shanghai, China). All the cellular and animal studies followed the local and federal regulations on cellular and animal studies. The animal studies were approved by the Ethics Committee at Sir Run Run Shaw Hospital at College of Medicine of Zhejiang University.

\section{Construction of a thin film by layer-by-layer assembly}

A gentamicin solution was prepared at a concentration of $200 \mu \mathrm{g}$ $\mathrm{mL}^{-1}$. OP-145 was dissolved in deionization (D.I.) water at a concentration of $200 \mu \mathrm{g} \mathrm{mL}{ }^{-1}$ with $0.15 \mathrm{M} \mathrm{NaCl}$ at $\mathrm{pH}$ 4.5. A glass or silicon substrate was cleaned with ethanol, soap, and DI water three times before a multilayer of thin films was deposited on it. To construct the thin films, the substrate was dipped into a gentamicin solution for $10 \mathrm{~min}$, followed by washing with DI water for $2 \mathrm{~min}$. The substrate was then soaked in the OP-145 solution for another $10 \mathrm{~min}$, followed by the same washing step. The deposition was repeated until a desired number of layers was constructed on the substrates.

\section{Film thickness characterization}

After depositing the desired number of layers of materials on the substrate, we dried the film with air. The film was then scratched with a razor blade. Profilometry (KLA-Tencor P-16 Profilometer) was used to measure the thickness at six different locations. Four to six different substrates were assessed to calculate the mean thickness.

\section{Release measurement}

For the release test, the substrates coated with films were stored at $4{ }^{\circ} \mathrm{C}$ before use. To measure the release profile of OP-145, films constructed with 30 layers of OP-145/gentamicin were soaked in $1 \times$ PBS solution, and the OP-145 peptide was labeled with rhodamine $\mathrm{B}$ isothiocyanate (RITC) molecules for release characterization at $560 \mathrm{~nm}$. To assess the release profile of gentamicin, 30 layers of OP-145/gentamicin were coated onto the substrate. The substrate was soaked in $1 \times$ PBS solution. The absorbance at $290 \mathrm{~nm}$ was measured by UV-vis spectroscopy for release assessment. The absorbance was converted into a mass unit for release assessment.

\section{Optical property assessment}

For optical clarity measurements, films with different numbers of layers were coated onto glass cover slips $(25 \mathrm{~mm} \times 25 \mathrm{~mm})$. Optical clarity was assessed by measuring the transmittance of light at $600 \mathrm{~nm}$ with an Evolution $60 \mathrm{UV}$-visible spectrophotometer (Thermo Scientific).

\section{Cell culture}

Cells were cultured in $\alpha$-MEM medium with $1 \%$ antibiotics (penicillin/streptomycin; Invitrogen, USA) and 10\% FBS. The cell culture conditions were kept at $5 \% \mathrm{CO}_{2}$ at $37^{\circ} \mathrm{C}$.

\section{Viability test}

For the viability study, cells were incubated at $37^{\circ} \mathrm{C}$ and washed with $1 \times$ PBS twice to remove the debris in the culture medium. 
The cells were then added to a mixture of $2 \mu \mathrm{M}$ calcein AM and 5 $\mu \mathrm{M}$ ethidium homodimer-1 (Life Technologies, UK) for live/ dead staining. The viability of the cells was assessed under an Olympus BX51 (Japan) fluorescence microscope.

\section{Metabolic activity test}

To measure metabolism quantitatively, Alamar blue solution was prepared by dissolving $425 \mathrm{mM}$ resazurin sodium salt (Sigma-Aldrich, Germany) in $1 \times$ PBS. The solution was then diluted by 10 times using the cell culture medium supplemented with 10\% FBS. The cells were cultured in 96-well plates, and $200 \mu \mathrm{L}$ Alamar blue solution was added to each well. A microplate reader (Fluoroskan Ascent FL; Thermo Fisher Scientific, USA) was used to assess the absorbance at $544 \mathrm{~nm}$ for metabolic activity measurement.

\section{In vivo model}

All animal procedures were performed in accordance with the Guidelines for Care and Use of Laboratory Animals of Zhejiang University and approved by the Animal Ethics Committee of College of Medicine at Zhejiang University. Mice were anesthetized with oxygen mixed with $2 \%$ isoflurane in a flow cabinet. The mice were injected with $0.05 \mathrm{mg} \mathrm{kg}^{-1}$ buprenorphine (Temgesic, RB Pharmaceuticals Limited) $15 \mathrm{~min}$ before the surgery to control the pain. The mice had their backs shaved and disinfected with $70 \%$ ethanol. A $12 \mathrm{~mm}$ incision was made on each mouse at a location $5 \mathrm{~mm}$ lateral to the spine. Silicone elastomer catheter segments $4 \mathrm{~mm}$ in length, with or without 30 layers of thin-film coating, were implanted into the incisions, and the incisions were closed with vicryl stitches (Ethicon). Next, $20 \mu \mathrm{L}$ of $S$. aureus in $0.9 \% \mathrm{NaCl}\left(4 \times 10^{5} \mathrm{CFU}\right.$ per $\left.\mathrm{mL}\right)$ was injected along the silicone elastomer. For the mice treated with a soluble mixture of OP-145 and gentamicin, the same loading of the cargos in PBS was injected in the same way into the mice. Mice with no treatment were used as a control. The mice were maintained in ventilated cages with food and water available at all times.

To test the level of in vivo infection, the mice were given oxygen mixed with $2 \%$ isoflurane, with buprenorphine used for pain control. Standard biopsies were taken from the implantation sites on day 7 . The mice were then euthanized by gradually exposing them to $\mathrm{CO}_{2}$ for 7-10 $\mathrm{min}$, followed by cervical dislocation. The $\mathrm{CO}_{2}$ displacement speed was controlled at around $5 \%$ for the first $2.5 \mathrm{~min}$ and increased to $20 \%$ for the rest 4.5 to $7.5 \mathrm{~min}$. All the biopsies contained subcutaneous tissues with the implants so that the infection level could be assessed quantitatively. The implants were rinsed with PBS for $1 \mathrm{~min}$ to remove nonadherent bacteria, followed by sonication in PBS for 3-5 min to dislodge adherent bacteria. Quantification was performed by measuring the number of bacteria in the PBS.

\section{Statistical analysis}

Statistical analyses were carried out using a one-way ANOVA with Tukey-Kramer post hoc analyses in MATLAB R2016b (Mathworks, Natick, MA, USA). A $p$ value $<0.05$ was used as the statistical significance level.

\section{Results}

We first measured the thickness and transparency of the antibacterial films. For antibacterial films with 1, 5, 10, and 30 bilayers, the thicknesses were 22, 109, 210, and $679 \mathrm{~nm}$, respectively. A linear relationship between the number of layers and the film thickness was observed (Fig. 1A). This is consistent with existing studies on layer-by-layer assembly technology in which a layer number-dependent film thickness was produced. We also measured the transparency of the film. Compared to the substrate with no films, the relative transparencies of the films with 10, 20, 30, and 40 layers were $89 \%, 77 \%, 71 \%$, and $67 \%$, respectively (Fig. 1B). No statistical difference was found between the 20 and 30 layered samples, which might be caused by the nature of the samples (Fig. 1B). There was a statistical difference between the empty substrate and the 20,30 , and 40 layers of film ( $p<0.05$ for all). However, the change in transparency did not affect the visibility of the substrate under the films, indicating that the addition of antibacterial films did not affect the transparency significantly. This information is vital because, in some in vitro studies or even in certain clinical transplant applications, it is necessary to visualize the morphology of biomaterial implants and observe the interaction between the materials and the surrounding tissues. A transparent film will permit these visualizations.

We next characterized the loading of the antibacterial components, measuring the impact of these films on cellular viability. The loadings of OP-145 in the films with 10, 20, 30, and 40 layers were $137.9,272.1,363.2$, and $483.0 \mu \mathrm{g}$, respectively. Similarly, the amounts of gentamicin in the films with 10, 20, 30 , and 40 layers were $41.3,83.1,117.9$, and $157 \mu \mathrm{g}$, respectively (Fig. 2A). Both components illustrated a linear relation between the number of layers and the cargo loading, which is also consistent with layer-by-layer assembly technology. In the PBS solution, both components were released continuously, showing a typically controlled release curve (Fig. 2B). The OP145 component was released slightly faster than the gentamicin, probably due to the charge and molecular size of the component (Fig. 2B). A controlled release of the components over 30 days indicated that these films could be employed for antibacterial functioning for a long time. This is a dramatic advantage compared to other biomaterials such as poly(lacticco-glycolic acid) (PLGA), for which a majority of the cargo was released within 24 hours.

Following the characterization study, we investigated the impact of antibacterial films on cell viability and cellular metabolic activity. For the multilayer structure, we prepared samples with 30 layers that was composed of OP-145 and gentamicin. As to the viability study, the use of OP-145 did not affect cell viability significantly (Fig. 3A). Gentamicin slightly reduced the cell viability. The antibacterial film did not affect the cell viability significantly, with an impact similar to the use of gentamicin in a soluble form (Fig. 3A). The co-use of gentamicin and OP-145 in a soluble form significantly affected the cell viability, probably due to the synergistic effect caused by the co-use of the two components $(p<0.05$, Fig. 3A). In the metabolic study, the use of 
A
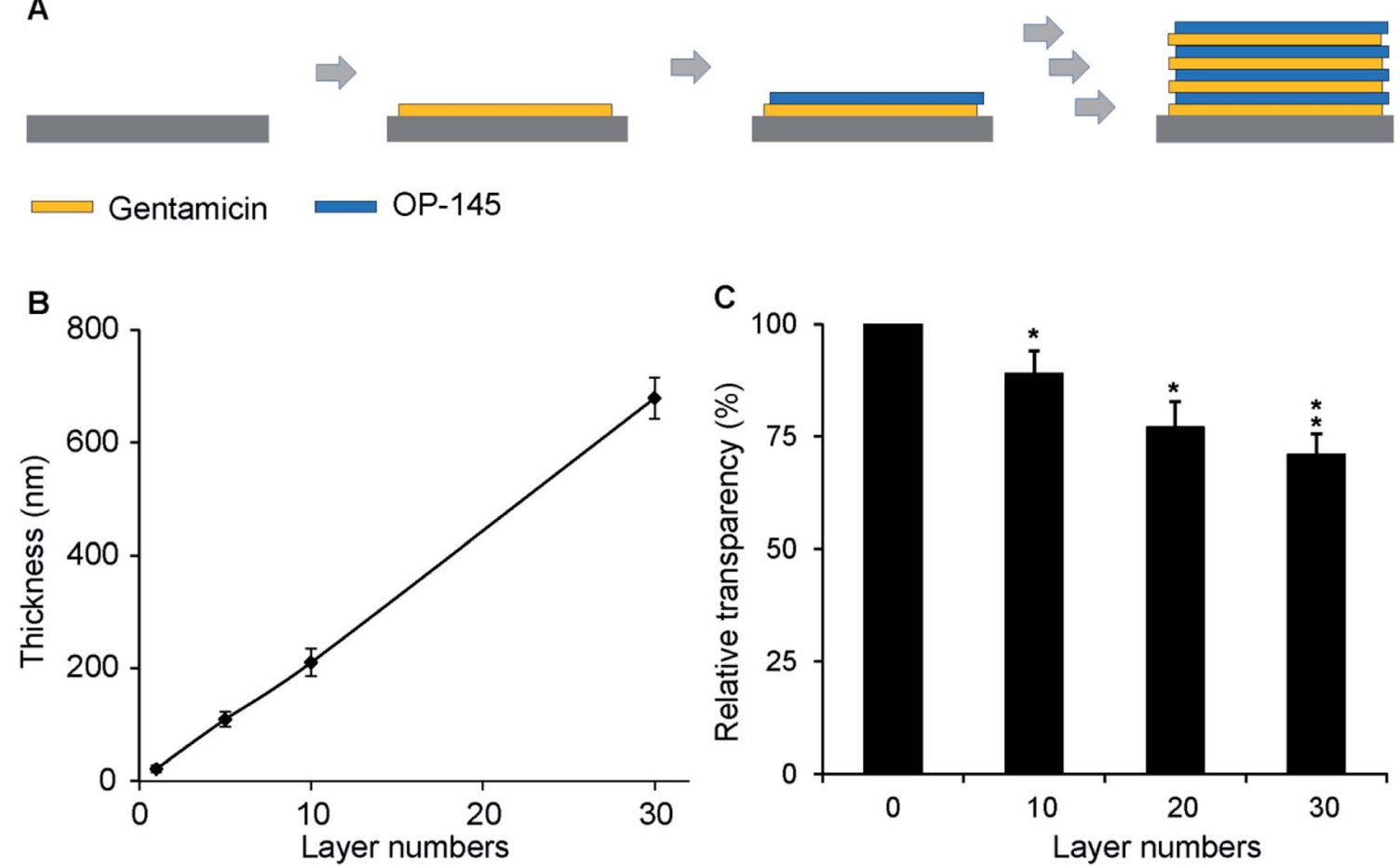

Fig. 1 Characterization of film thickness and transparency. (A) Schematic picture showing the construction of thin film composed of gentamicin and OP-145 with (B) thicknesses of the films with different numbers of layers (i.e., 1, 5, 10, and 30 layers). (C) Relative transparencies of the films with different numbers of layers (as in A). Each layer contained an gentamicin/OP-145 bilayer. The thickness increased with the number of layers. The addition of each extra layer slightly affected the transparency of the films. * $p<0.05, * * p<0.01$.

gentamicin reduced the metabolic activity of the cells (Fig. 3B). Similarly, the co-use of soluble gentamicin and OP-145 significantly decreased the cellular metabolic activity ( $p<0.05$, Fig. 3B). The OP-145 component did not affect cellular metabolic activity. The use of 30-layer antibacterial films did not dramatically affect metabolic activity, either (Fig. 3B). This is probably because the controlled release of gentamicin reduced the impact of this cargo on the metabolism.
We next assessed whether the antibacterial films were able to resist S. aureus biofilm formation. After 36 hours, S. aureus formed biofilms on the uncoated and plasma-coated microtiter plates. However, both OP-145 and gentamicin were able to resist fast $S$. aureus biofilm formation. OP- 145 allowed $53.1 \%$ and $47.2 \%$ biofilm formation on the uncoated and plasma-coated substrates. Gentamicin allowed $45.2 \%$ and $47.9 \%$ biofilm formation on the uncoated and plasma-coated microtiters. These data indicate that
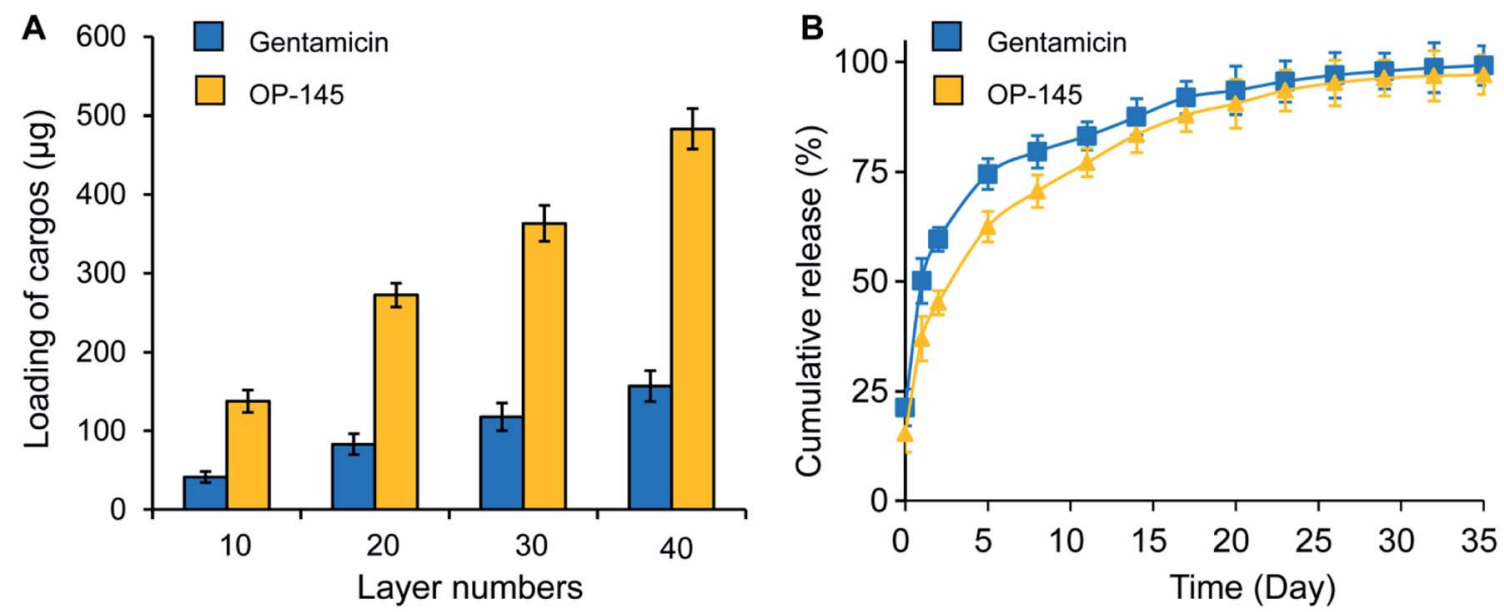

Fig. 2 Assessment of cargo loading and cumulative release from the thin films. (A) Loading of OP-145 (yellow bar) and gentamicin (blue bar) in the thin films with different numbers of layers (i.e., 10, 20, 30, and 40 layers). (B) Cumulative release of OP-145 (yellow bar) and gentamicin (blue bar) from a thin film with 30 layers of material. The cargos were released over 30 days. 

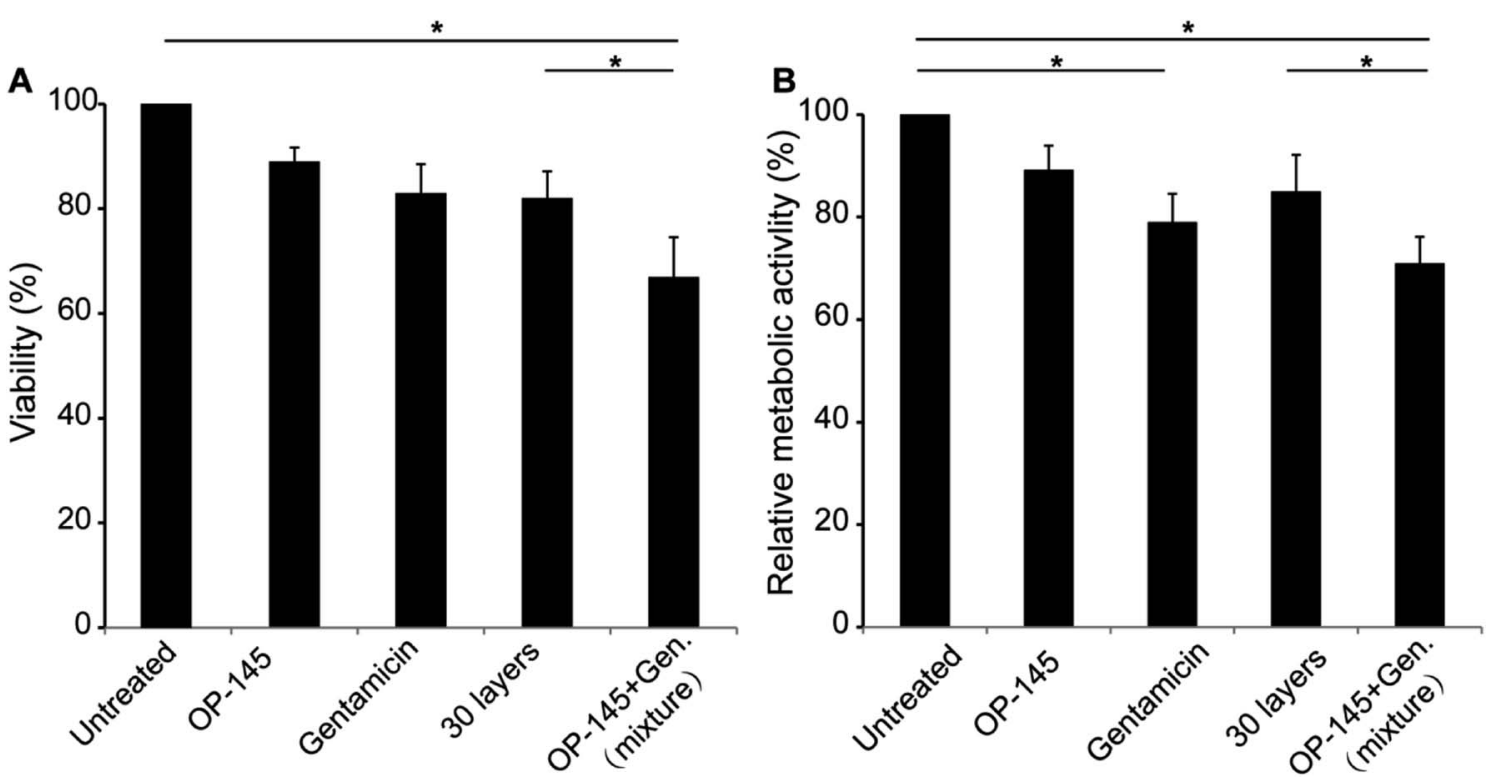

Fig. 3 Impact of each cargo on cell viability and metabolic activity. (A) Impact of OP-145 and gentamicin, either in the soluble form or in film layers, on cell viability. (B) The influence of OP-145 and gentamicin, either in the soluble form or in film layers, on cellular metabolic activity. Thin films containing 30 layers of OP-145/gentamicin were used for the test. The OP-145 + gentamicin solution contained the same amount of cargo as the film with 30 layers.

both therapeutic cargos were able to resist $S$. aureus formation. The co-use of OP-145 and gentamicin also reduced the formation of biofilm, allowing $37 \%$ and $35.2 \%$ biofilm formation on the substrates and demonstrating a synergistic effect of the two cargos (Fig. 4). For the antibacterial films with 30 layers, the levels of biofilm formation on the uncoated and plasma-coated substrates were $12.8 \%$ and $11.5 \%$, respectively. Compared to using soluble OP-145 or gentamicin or the two soluble cargos together, there was a dramatic decrease in the formation of biofilm in the samples coated with 30 layers of film, indicating greater antibacterial effects of the multilayer thin films.

Next, we studied the in vivo antibacterial activity of the thin films with a mouse subcutaneous implant infection model. The study tested the in vivo colonization of $S$. aureus on silicone elastomer implants. OP-145 and gentamicin were assembled onto the elastomer implants as thin films. A soluble mixture of an equal amount of OP-145 and gentamicin was used as a control. Another control was the untreated mice. The $\log \mathrm{CFU}$ values in the untreated group, the soluble mixture group, and the thin film group were 4.87, 3.02, and 1.96, respectively (Fig. 5A). The soluble mixture of OP-145 and gentamicin reduced the bacterial progression significantly compared to the untreated group ( $p<$ 0.05 , Fig. 5B). The OP-145/gentamicin thin film caused a significant reduction in the bacteria on the implants ( $p<0.05$, Fig. 5B). Comparing the soluble mixture group with the thin film group, there was also a statistical difference in the inhibition of the in vivo bacterial progression, indicating greater effectiveness of the OP-145/gentamicin thin film ( $p<0.05$, Fig. 5B).

\section{Discussion}

Existing technologies have mainly focused on generating antiadhesive surfaces or coating antibacterial components onto implants to prevent potential S. aureus infections. ${ }^{26}$ Although these technologies can partially prevent clinical bacterial contamination, they have multiple drawbacks. For example, anti-adhesive surfaces can prevent the colonization of bacteria on implants, but they cannot prevent the growth of bacteria in the tissues implanted with the materials or devices. ${ }^{27-29}$ For antibacterial coating technologies, the release of therapeutic components usually lasts for a short period of time, and only a subinhibitory dose of cargos is released, which cannot generate a long-lasting therapeutic effect that inhibits a broad range of bacteria. ${ }^{30}$ In this work, we showed that the

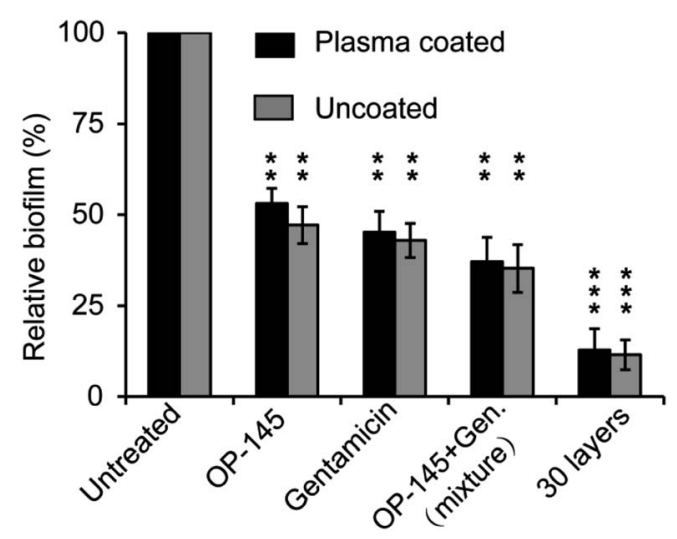

Fig. 4 The influence of cargo, either in the form of a thin film or a cargo mixture or soluble cargo alone, on the formation of biofilm. Two types of microtiter (uncoated and plasma-coated) were adopted for the test. Thin films that contained 30 layers of OP-145/gentamicin were employed for the test. The soluble OP-145 + gentamicin contained the same amount of cargo as the film with 30 layers. $* p<0.05$, $* * p<0.01$, and $* * * p<0.001$. The statistical analysis compared treated groups against the untreated group. 



Fig. 5 Assessment of the in vivo antibacterial activity of the thin film in a mouse subcutaneous implant infection model. (A) The value of the log CFU per sample in each mouse in each group. (B) The average log CFU per sample was measured in three groups: untreated mice (i.e., mice inoculated with the bacteria), mice treated with a soluble mixture of OP-145 + gentamicin, and mice treated with implants coated with 30 layers of OP-145/gentamicin. The soluble mixture and the thin film had the same loading of cargo. * $p<0.05, * * p<0.01$.

simultaneous use of OP-145 and gentamicin in polyelectrolyte multilayers prevents $S$. aureus infections. This co-delivery technology can generate a potent synergistic antibacterial effect. For example, existing studies have reported the isolation of gentamicin-resistant bacteria from patients treated with gentamicin. ${ }^{26,29}$ This bacterial resistance effect may require the use of other antibacterial agents, such as peptides that have antibacterial effects. For enhanced antibacterial function, the co-delivery of multiple therapeutic components in multilayered structures may generate even more potent antibacterial effects. $^{26,29}$

In this work, we have illustrated the simultaneous delivery of two therapeutic components to prevent $S$. aureus infections in implanted biomaterials. A major advantage of this technology is that it uses the synergistic effects of two antibacterial cargos, which is more effective than using a single component. Considering the fact that a broad range of antibiotic drugs are losing their efficacy in preventing bacteria effectively, the synergistic use of multiple antibiotic components is highly desirable in preventing biomaterial-related $S$. aureus infections. At the same time, another advantage of this technology is the multilayer structure demonstrated in this study. A multilayer structure built with a layer-by-layer assembly technique allows the incorporation of several therapeutic cargos into the functional thin films. Here, we used only two types of antibacterial components. However, this technology actually allows more than two types of functional cargo to be employed, greatly enhancing the potential therapeutic efficacy of this technology. A further advantage of this multilayer antibacterial film is that the technique enables the control of the relative ratios of the therapeutic components in the thin film, making this antibacterial design more flexible. Also, the multilayer structure allows the prolonged release of the antibacterial components. This feature is highly useful because it enables long-lasting antibacterial effects, which prevent potential bacterial infections during the whole implantation time.
In our work, several points regarding the characterization of thin films, the in vitro and the in vivo study deserve a brief discussion as well. For example, in Fig. 1B, there was a difference between 20 and 30 layered samples, but no statistical difference was identified. This might be caused by the nature of these samples. Additionally, the profilometry for the thickness characterization was used in a "contact" mode, with a stylus force around 1-50 mg, as indicated by the producer. We need to mention that our current characterization by using the profilometry cannot avoid the potential friction impact. However, of more important is that the profilometry cauterization indicated the successful buildup of multilayer structure in the thin films. As to the in vitro and in vivo study, we observed a difference in the anti-bacterial efficacy, as illustrated by efficacy variation between Fig. 5 (i.e., in vivo) and Fig. 3/Fig. 4 (i.e., in vitro). The difference might be caused by the difference between the in vitro and in vivo environment, where the in vivo environment is more complicated. In Fig. 3, the study showed that the use OP145 and gentamicin impacted cellular viability more than using 30 layered samples composed of OP-145 and gentamicin. The thin film caused less toxicity to cells in vitro probably because the controlled release of OP-145 and gentamicin as illustrated in Fig. 2. As to the in vitro study, we need to mention that the cell medium has multiple components that may potential interact with the peptide as well as gentamicin. Our current in vitro study did not investigate the impact of cell culture medium, but it does not necessarily mean that this potential impact does not exist. In Fig. 5, the thin film was more effective in inhibiting bacterial infection probably due to a similar reason. However, both a long lasting antibacterial effect and a minimum toxicity were desirable in the clinical application of these cargos. Thus the data in vitro and in vivo study together illustrated that we could minimize the toxicity of OP-145 and gentamicin while achieving a prolonged antibacterial effect by using the multilayer structure. These in vitro and in vivo data indicated that a prolonged antibacterial effect with minimized toxicity issue could be achieved by using a layered structure. 
Technically, this work used both in vitro and in vivo models to test the antibacterial effects of multilayer thin films. These studies, along with others' work, have illustrated well the potency and effectiveness of the films, but more studies are needed to fully explore the effectiveness of this technology. ${ }^{31,32}$ For example, a prolonged study may be required in our future work to observe the antibacterial effect of this technique over the whole implantation time. Also, future studies may include investigations of the impact of using varied ratios of the two cargos, where the goal is to reach a maximal antibacterial effect and a minimum toxicity. Overall, future work requires looking into more details than just the simple in vitro and in vivo models.

\section{Conclusion}

In this study, we designed antibacterial films assembled from OP-145 and gentamicin, which are antibacterial components that can be employed to resist $S$. aureus in implanted biomaterials. The study illustrated a linear increase in the layer thickness and cargo loading in the thin film and demonstrated a controlled release of the cargos over 30 days. In the viability study, compared to soluble gentamicin, the thin films did not affect cell viability and reduced the impact of gentamicin on cellular metabolism. In the antibacterial study, the thin films were able to resist biofilm formation more effectively than the soluble cargos separately or together. The study demonstrated how assembling OP-145 and gentamicin into thin films can reduce potential $S$. aureus infections in implanted biomaterials. In vivo, the OP-145/gentamicin thin film can inhibit bacterial progression more effectively than an equal amount of a soluble mixture of OP-145 and gentamicin.

\section{Funding}

This work was supported by the China Natural Science Foundation (2017A610245) and Key Discipline of Ningbo (2016018).

\section{Availability of data and materials}

All relevant data and supporting information files are contained within the paper.

\section{Ethics approval and consent to participate}

All the cellular and animal studies followed the local and federal laws and regulations.

\section{Patient consent for publication}

Not applicable.

\section{Conflicts of interest}

The authors have no conflicts of interest.

\section{References}

1 R. O. Darouiche, N. Engl. J. Med., 2004, 350, 1422-1429.

2 S. Nishimura, T. Tsurumoto, A. Yonekura, K. Adachi and H. Shindo, J. Orthop. Sci., 2006, 11, 46-50.

3 C. P. Duncan and C. Beauchamp, Orthop. Clin. N. Am., 1993, 24, 751-759.

4 M. Riool, L. de Boer, V. Jaspers, C. M. van der Loos, W. J. B. van Wamel, G. Wu, P. H. S. Kwakman and S. A. J. Zaat, Acta Biomater., 2014, 10, 5202-5212.

5 D. R. Murdoch, S. A. Roberts, V. G. Fowler Jr, M. A. Shah, S. L. Taylor, A. J. Morris and G. R. Corey, Clin. Infect. Dis., 2001, 32, 647-649.

6 P. Sendi, F. Banderet, P. Graber and W. Zimmerli, J. Infect., 2011, 63, 17-22.

7 S. Bakhshandeh, Z. Gorgin Karaji, K. Lietaert, A. C. Fluit, C. H. E. Boel, H. C. Vogely, T. Vermonden, W. E. Hennink, H. Weinans, A. A. Zadpoor and S. Amin Yavari, ACS Appl. Mater. Interfaces, 2017, 9, 25691-25699.

8 A. de Breij, M. Riool, P. H. Kwakman, L. de Boer, R. A. Cordfunke, J. W. Drijfhout, O. Cohen, N. Emanuel, S. A. Zaat, P. H. Nibbering and T. F. Moriarty, J. Controlled Release, 2016, 222, 1-8.

9 J. S. Moskowitz, M. R. Blaisse, R. E. Samuel, H. P. Hsu, M. B. Harris, S. D. Martin, J. C. Lee, M. Spector and P. T. Hammond, Biomaterials, 2010, 31, 6019-6030.

10 J. M. Andrews, J. Antimicrob. Chemother., 2001, 48(suppl 1), 5-16.

11 R. E. Isturiz and C. Carbon, Infect. Control Hosp. Epidemiol., 2000, 21, 394-397.

12 I. N. Okeke, Resistance Phenomenon in Microbes and Infectious Disease Vectors, 2003, pp. 132-139.

13 N. Malanovic, R. Leber, M. Schmuck, M. Kriechbaum, R. A. Cordfunke, J. W. Drijfhout, A. de Breij, P. H. Nibbering, D. Kolb and K. Lohner, Biochim. Biophys. Acta, 2015, 1848, 2437-2447.

14 J. Turner, Y. Cho, N. N. Dinh, A. J. Waring and R. I. Lehrer, Antimicrob. Agents Chemother., 1998, 42, 2206-2214.

15 P. Jorge, A. Lourenco and M. O. Pereira, Biofouling, 2012, 28, 1033-1061.

16 S. C. Park, Y. Park and K. S. Hahm, Int. J. Mol. Sci., 2011, 12, 5971-5992.

17 Z. Gong, S. P. Ikonomova and A. J. Karlsson, Protein Sci., 2018, 27, 702-713.

18 C. L. Romano, S. Scarponi, E. Gallazzi, D. Romano and L. Drago, J. Orthop. Surg. Res., 2015, 10, 157.

19 J. Gallo, M. Holinka and C. S. Moucha, Int. J. Mol. Sci., 2014, 15, 13849-13880.

20 S. Pahal, R. Gakhar, A. M. Raichur and M. M. Varma, IET Nanobiotechnol., 2017, 11, 903-908.

21 T. Boudou, T. Crouzier, K. Ren, G. Blin and C. Picart, Adv. Mater., 2010, 22, 441-467.

22 B. Wang, T. Jin, Q. Xu, H. Liu, Z. Ye and H. Chen, Bioconjugate Chem., 2016, 27, 1305-1313.

23 M. C. Berg, L. Zhai, R. E. Cohen and M. F. Rubner, Biomacromolecules, 2006, 7, 357-364. 
24 B. Y. Li, B. B. Jiang, B. M. Boyce and B. A. Lindsey, Biomaterials, 2009, 30, 2552-2558.

25 P. Schultz, D. Vautier, L. Richert, N. Jessel, Y. Haikel, P. Schaaf, J. C. Voegel, J. Ogier and C. Debry, Biomaterials, 2005, 26, 2621-2630.

26 G. Batoni, G. Maisetta and S. Esin, BBA, Biochim. Biophys. Acta, Biomembr., 2016, 1858, 1044-1060.

27 B. G. Zhang, D. E. Myers, G. G. Wallace, M. Brandt and P. F. Choong, Int. J. Mol. Sci., 2014, 15, 11878-11921.
28 F. Pishbin, V. Mourino, S. Flor, S. Kreppel, V. Salih, M. P. Ryan and A. R. Boccaccini, ACS Appl. Mater. Interfaces, 2014, 6, 8796-8806.

29 C. D. Chaput, Spine, 2017, 42(suppl 7), S32.

30 D. Neut, H. van de Belt, I. Stokroos, J. R. van Horn, H. C. van der Mei and H. J. Busscher, J. Antimicrob. Chemother., 2001, 47, 885-891.

31 X. Y. Zhu and X. J. Loh, Biomater. Sci., 2015, 3, 1505-1518.

32 T. He and V. Chan, J. Biomed. Mater. Res., Part A, 2010, 95a, 454-464. 